\title{
Interaction dans la Vallée
}

\author{
Diane Brassard et Marc-Urbain Proulx
}

Centre de recherche sur le développement territorial (CRDT) de l'UQAC

À l'ère de ladite économie du savoir, la compétitivité des entreprises et des nations passe largement par l'innovation sous la forme de nouveaux procédés de production, de nouveaux produits, de nouveaux services, de nouveaux marchés. L’innovation représente le moteur de la dynamique socio-économique contemporaine. Dans sa plus simple expression, elle se définit telle une «nouvelle combinaison de facteurs $»$.

Figure 1

Les segments du modèle linéaire classique de l'innovation

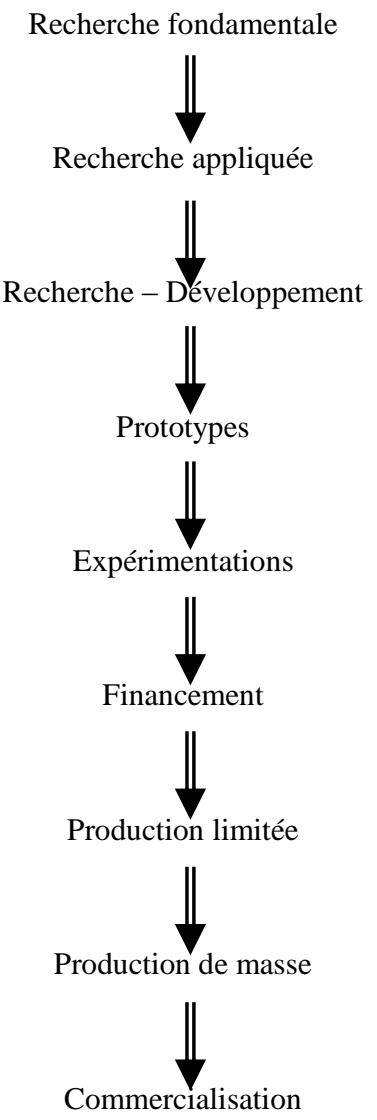


Le modèle explicatif classique de l'innovation qu'on associe à Joseph Schumpeter adopte une forme linéaire. Il est représenté telle une séquence d'étapes distinctes effectuées en filiation (figure 1). Différents segments d'une filière génératrice d'innovation logent ainsi les facteurs à combiner d'une manière nouvelle.

Selon cette conception schumpétérienne, on considère deux impulsions fondamentales qui agissent pour stimuler le processus d'innovation, soit la technologie en amont et le marché en aval. Pour qu'une société soit innovatrice, il faut que ces impulsions soient au rendez-vous pour alimenter les divers segments du processus.

Ainsi selon ce modèle, l'innovation apparait généralement autour des grands laboratoires de recherche et des services supérieurs spécialisés largement localisés au sein des grands marchés urbains. Une fois mis au point, les nouveaux produits, services et procédés de production se diffusent dans les périphéries. À cet effet, différentes modalités de diffusion furent dans le passé proposées, testées et validées, notamment la diffusion radiale en tache d'huile par couronnes successives autour des centres urbains et aussi celle de nature hiérarchique, descendante par échelles, vers les centres secondaires, tertiaires, quaternaires.

Aussi pertinent qu'il soit, ce modèle linéaire ne peut cependant réclamer un caractère universel dans le contexte contemporain par lequel l'innovation s'avère généralisée à l'ensemble des activités économiques, sociales, culturelles et institutionnelles. Valable certes, cette simplicité linéaire ne résiste pas à la complexité des faits dans les processus réels. En réalité, deux caractéristiques du modèle classique de l'innovation sont désormais questionnées de front, soit la linéarité amont - aval du processus ainsi que les modalités de diffusion.

Figure 2

\section{Modèle interactif de l'innovation}

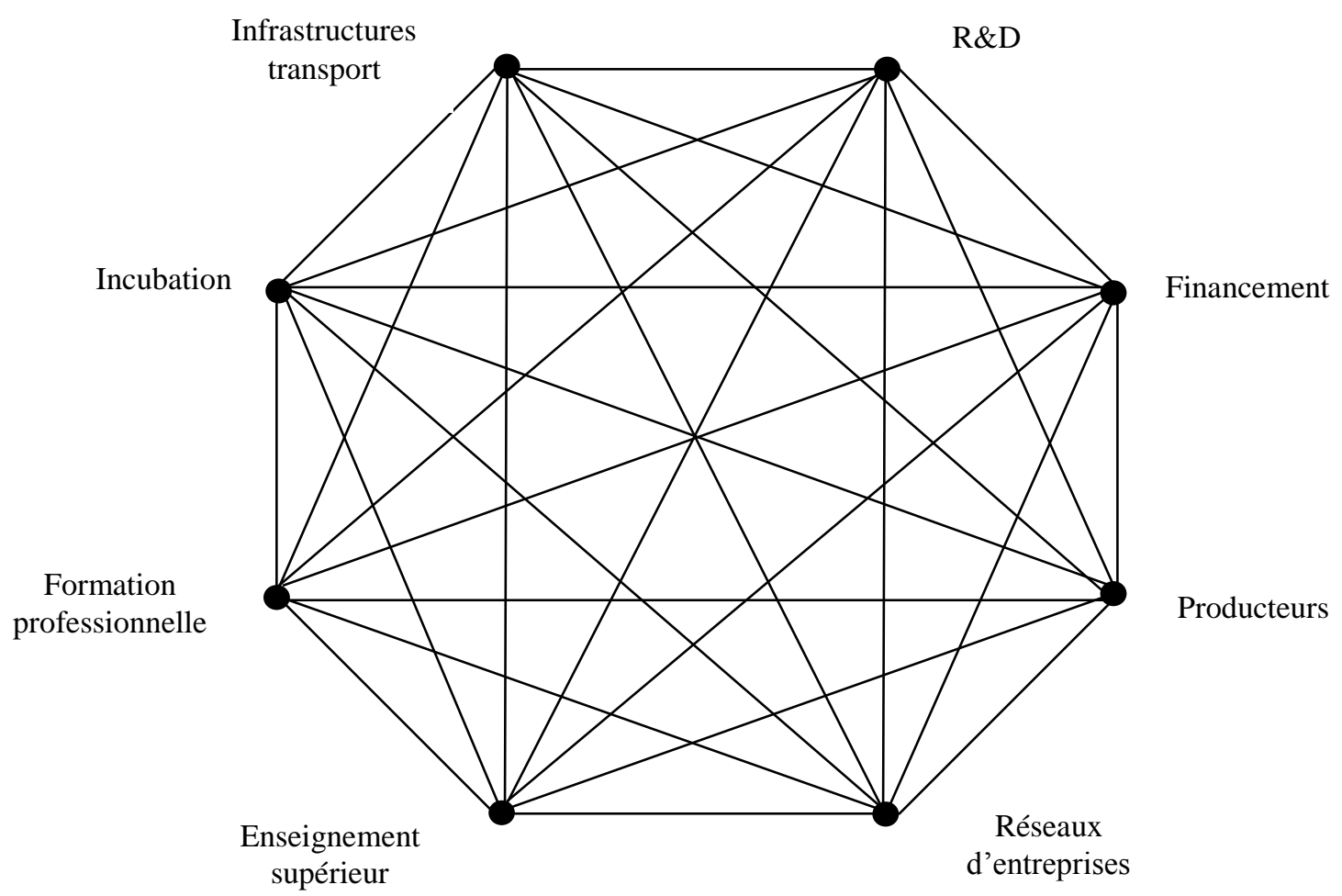


De fait, un nouveau modèle général de nature «interactive» s'avère d'une très grande pertinence actuellement pour expliquer les processus de l'innovation (figure 2). Celui-ci illustre le rôle primordial et central de ladite conception fertilisée à partir des facteurs. Conception de produits et de procédés qui découle bien sûr de la perception par les acteurs d'occasions du marché en liaison avec des possibilités techniques, tout en la dépassant néanmoins dans une fonction fondamentalement relationnelle, dynamique, cumulative, cognitive et évolutive entre les divers acteurs concernés. Selon cette modélisation, la hiérarchie et le marché jouent un rôle moindre qu'on le croyait dans le processus. De fait, les relations interactives génératrices d'innovations épousent largement d'autres mécanismes institutionnels qui soutiennent les activités d'apprentissage collectif et de créativité.

Selon cette approche explicative, le phénomène de l'innovation s'avère moins dépendant de l'impulsion en aval et en amont illustrée par le modèle linéaire, soit à partir de la recherche d'un côté et de la présence d'un marché de l'autre. Les segments intermédiaires et leurs acteurs seraient aussi très importants, voire essentiels dans le processus interactif. En réalité, l'émergence de l'innovation s'inscrit souvent sous la forme d'une «fertilisation croisée » entre différents segments de la filière en interaction créatrice. La linéarité du processus fait alors place à un jeu relationnel composé de retours, de sauts derrière, devant ou sur place, par lesquels l'apprentissage collectif des acteurs devient central dans la combinaison appropriée des facteurs.

\section{Le système productif de la Vallée}

Selon le «Profil de la recherche au Saguenay - Lac-Saint-Jean » publié en 2005 par Samson Bélair / Deloitte \& Touche, le système
Dans cet esprit d'interaction et d'apprentissage collectif à l'innovation, le territoire semble jouer en de nombreux endroits, souvent relativement périphériques aux grands centres urbains, un rôle de creuset pour favoriser la fertilisation croisée entre les acteurs appartenant aux divers segments. Les exemples sont légion à cet effet dans la plupart des pays, notamment les fameuses zones industrielles à succès qu'on retrouve en Italie, en France, en Allemagne, aux ÉtatsUnis, en Chine, au Brésil. En réalité dans cette nouvelle modélisation du processus de l'innovation, le territoire plus ou moins périphérique se voit doté non seulement de la traditionnelle fonction de captage des impulsions technologiques et mercantiles à partir des grands centres urbains, mais aussi bel et bien d'une fonction de « fertilisation par l'interaction » favorisée par la proximité entre les acteurs qui détiennent les facteurs à combiner (figures 1 et 2).

Sur la base de ce modèle, nous avons effectué une observation et une analyse de l'interaction au sein de la Vallée de l'aluminium au Québec. Pour tenter de comprendre comment s'articule l'interaction dans la Vallée, nous présenterons les principaux mécanismes qui favorisent la mise en relations entre les diverses organisations concernées. Notre période de référence s'étend du $1^{\text {er }}$ janvier 2005 au 31 mars 2008. Nous ferons ensuite le portrait de chacun des mécanismes interactifs retenus: les regroupements, les petits événements, les réseaux et les catalyseurs du milieu. En conclusion, nous verrons les forces et les faiblesses du réseautage dans le domaine de l'aluminium dans la région et quelques pistes de solutions pour améliorer la situation.

productif de la Vallée de l'aluminium comprend d'abord les matières premières et l'aluminium primaire, d'où découlent trois 
grands volets et toutes leurs composantes respectives, soit la coulée de lingots, la coulée continue et la fonderie. Les produits finis et semi-finis complètent le tout, que ce soit dans le domaine du transport, de la construction ou des biens de consommation. On signifie ici l'ensemble des entreprises régionales impliquées dans le secteur de l'aluminium, y compris les équipementiers. Selon le modèle interactif de l'innovation proposé ci-dessus, d'autres composantes (figure 3) gravitent autour de ces producteurs de la Vallée de l'aluminium, notamment les centres de R\&D, la formation professionnelle, l'enseignement supérieur, les organisations de soutien, les gestionnaires d'infrastructures, les organismes de financement.

Figure 3

\section{Principales composantes du système productif de la Vallée}

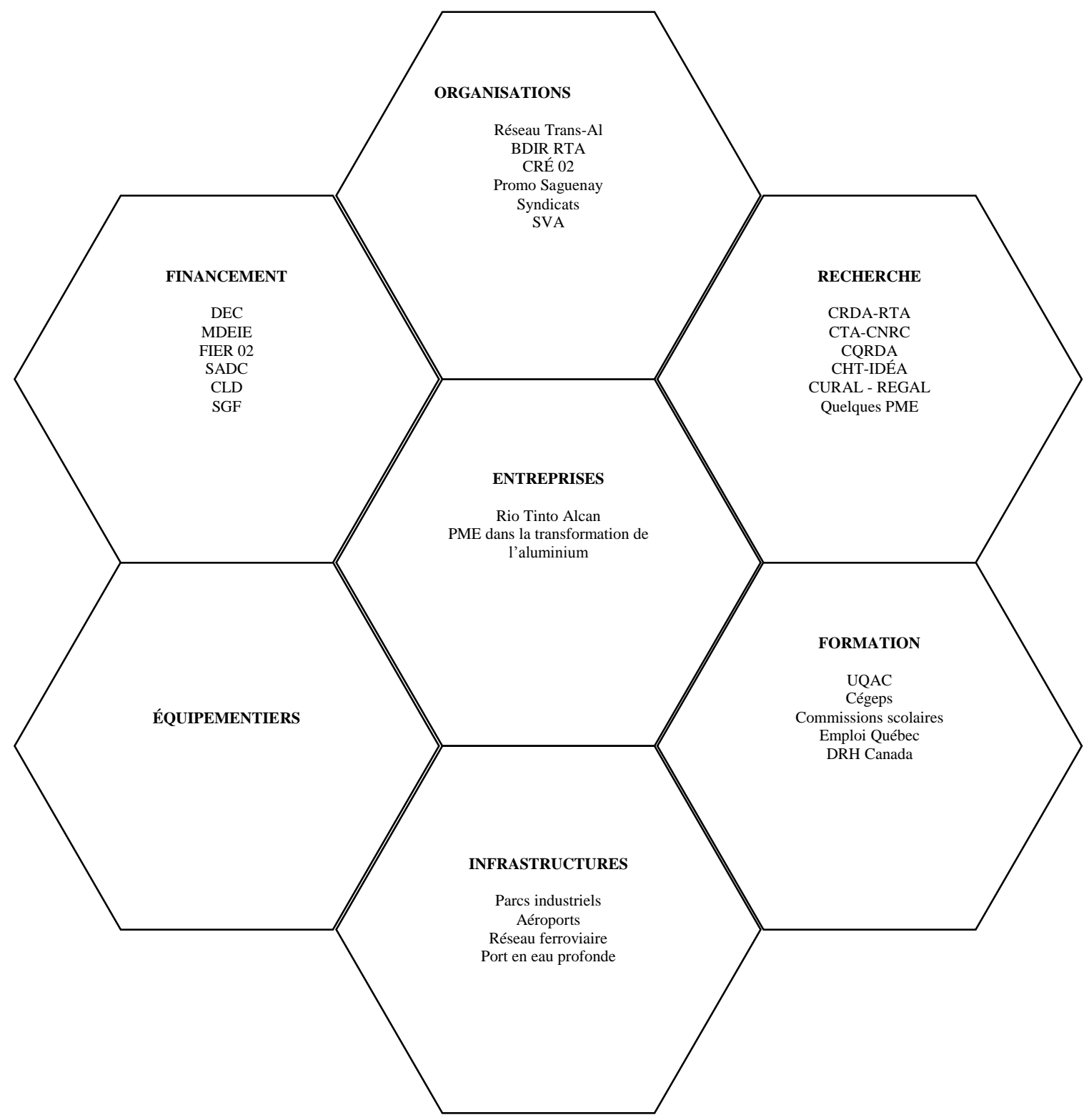


Pour observer les champs d'interaction entre les partenaires de l'innovation dans la Vallée, nous avons exploré différentes sources de données, dont les journaux locaux (Le Quotidien, Progrès-Dimanche, Journal du LacSaint-Jean, le Courrier du Lac et l'Étoile du Lac), tous disponibles sous forme électronique à la Bibliothèque de l'UQAC - Banque de données nommée «Biblio-branchée », ainsi que le Bulletin régional (LBR) Saguenay Lac-Saint-Jean également disponible sur internet $^{1}$. Nous sommes conscients que certains événements non médiatisés ont

\section{Les regroupements formels}

L'interrogation de la banque de données «Biblio-branchée » de l'UQAC permet de repérer plus d'une vingtaine de regroupements reliés au domaine de l'aluminium, dont $75 \%$ s'y consacre en exclusivité. Il s'agit d'associations, de syndicats, de groupes sociaux ou d'intérêt et divers comités. À ces regroupements recensés, il faudrait évidemment ajouter éventuellement les différents Conseils et Commissions du secteur public dont les activités concernent plus ou moins les enjeux reliés à l'innovation dans la Vallée. Soulignons notamment le Conseil municipal de Saguenay, la Commission scolaire des Rives-du-Saguenay, les conseils d'administration des organismes de développement comme les CLD, les SADC, Promotion Saguenay.

Le réseau régional de RTA principalement impliqué dans le secteur primaire s'avère évidemment bien présent. On compte aussi sur la présence de la SVA ainsi que du Réseau Trans-Al regroupant plusieurs PME. Bien actifs comme regroupements, il faut compter sur les quatre syndicats du secteur de l'aluminium. Le Regroupement des équipementiers s'avère aussi bel et bien sûrement échappé à notre observation incomplète pendant la période considérée.

Nous avons aussi consulté les sites internet des différentes organisations impliquées dans la Vallée, notamment Rio Tinto Alcan (RTA), la SVA, le Réseau Trans-Al, la CRÉ 02, la SFR 02, les centres de recherche (CTA-CRNC, CHT-IDÉA, CQRDA, REGAL, CURAL) ainsi que les organisations de la FORMATION (UQAC, cégeps, commissions scolaires, formation professionnelle) et du FINANCEMENT (DEC, MDEIE, FIER 02, SADC, CLD, SGF).

présent. Du côté de la recherche, le CURAL et le RÉGAL représentent deux regroupements importants. Il faut aussi ajouter le Comité d'orientation de la carte routière technologique canadienne de la transformation de l'aluminium (édition 2006), le Comité d'implantation du Centre de valorisation d'Arvida et d'aluminium dans l'Église Saint-Jacques et le Comité pour une aluminerie dans le Comté de Roberval mis en place en 2007. Existent aussi deux groupes dans la formation, soit le Comité «Rêver l'aluminium » et le Comité sectoriel de main-d'œuvre de la métallurgie.

Le tableau 1 présente nos observations à propos des liens que tous ces regroupements entretiennent avec les différentes composantes du système productif de la Vallée de l'aluminium, du moins ceux qui ont fait l'objet d'au moins un article dans les journaux locaux au cours de la période. Globalement, quatre regroupements se distinguent pour le nombre de relations tissées, soit le Réseau Trans-Al, le Comité sectoriel de main-d'œuvre de la métallurgie, le réseau régional Rio Tinto Alcan et le Comité d'orientation de la carte routière technologique canadienne. 
Tableau 1

Liaisons entre les regroupements et les composantes de la Vallée

\begin{tabular}{|c|c|c|c|c|c|c|c|c|c|c|}
\hline \multirow[b]{2}{*}{ REGROUPEMENT SELON LEUR COMPOSANTE PRINCIPALE } & \multicolumn{10}{|c|}{ EN LIEN AVEC LES AUTRES COMPOSANTES } \\
\hline & 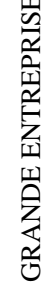 & $\sum_{2}^{N}$ & 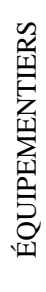 & 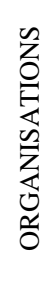 & 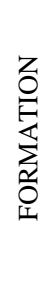 & 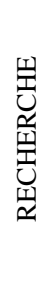 & 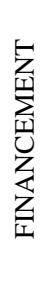 & 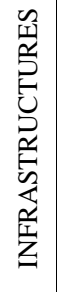 & 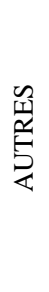 & 造 \\
\hline Rio Tinto Alcan & & $\mathrm{X}$ & $\mathrm{X}$ & & $\mathrm{X}$ & $\mathrm{X}$ & & & & 4 \\
\hline Société de la vallée de l'aluminium & & & & & $\mathrm{X}$ & $\mathrm{X}$ & $\mathrm{X}$ & & & 3 \\
\hline Réseau Trans-Al & $\mathrm{X}$ & $\mathrm{X}$ & & & $\mathrm{X}$ & $\mathrm{X}$ & $\mathrm{X}$ & & & 5 \\
\hline Syndicat national des employés de l'aluminium d'Arvida & $\mathrm{X}$ & & & & & & & & & 1 \\
\hline Syndicat des travailleurs de l'aluminium d'Alma & $\mathrm{X}$ & & & & & & & & & 1 \\
\hline Syndicat des employés des installations portuaires & $\mathrm{X}$ & & & & & & & $\mathrm{X}$ & & 2 \\
\hline Syndicat des employés d'énergie électrique Québec & $\mathrm{X}$ & & & & & & & $\mathrm{X}$ & & 2 \\
\hline Regroupement des équipementiers du SLSJ & $\mathrm{X}$ & $\mathrm{X}$ & & & & & & & & 2 \\
\hline Comité « Rêver l'aluminium » & & $\mathrm{X}$ & & $\mathrm{X}$ & & & & & & 2 \\
\hline Comité sectoriel de main-d'œuvre de la métallurgie & $\mathrm{X}$ & $\mathrm{X}$ & & $\mathrm{X}$ & & $\mathrm{X}$ & $\mathrm{X}$ & & & 5 \\
\hline CURAL & $\mathrm{X}$ & & & $\mathrm{X}$ & $\mathrm{X}$ & & & & & 3 \\
\hline REGAL & $\mathrm{X}$ & & & $\mathrm{X}$ & $\mathrm{X}$ & & & & & 3 \\
\hline Comité de la carte routière technologique canadienne & $\mathrm{X}$ & & & $\mathrm{X}$ & & $\mathrm{X}$ & $\mathrm{X}$ & & & 4 \\
\hline Comité du Centre de valorisation d'Arvida & $\mathrm{X}$ & & & $\mathrm{X}$ & & & & & & 2 \\
\hline Comité Aluminerie dans le Comté de Roberval & & & & $\mathrm{X}$ & & & & & & 1 \\
\hline TOTAL & 11 & 5 & 1 & 7 & 5 & 5 & 4 & 2 & $\mathbf{0}$ & 40 \\
\hline
\end{tabular}

Source : Compilation personnelle à partir de la banque de données Biblio-branchée de l'UQAC

Notre observation nous permet d'identifier d'autres regroupements dont l'implication s'avère plus ponctuelle dans le domaine de l'aluminium. Soulignons notamment la CRÉ 02, par l'entremise de son Comité de maximisation des retombées économiques, la

\section{Les petits événements}

$\mathrm{Au}$ cours de la période retenue, nous avons répertorié un total de 112 petits événements en lien avec le domaine de l'aluminium. Les petits événements sont des activités dont la durée ne dépasse par un mois. Ils rassemblent des gens dans un lieu désigné afin de favoriser l'échange des idées et le réseautage sur une
SFR 02, la Commission Gagné qui s'est penchée sur les aides fiscales consenties aux régions ressources, le Comité d'investisseurs de FIER 02, le Regroupement des Chambres de commerce, le Regroupement des CLD, le Comité de diversification industrielle. base informelle. Ils prennent des formes très variées, de journées thématiques à des visites industrielles, en passant par des galas, des audiences publiques et des salons thématiques.

Nous avons noté un essor important des petits événements au cours du quatrième trimestre de 
2006, autour de l'annonce publique de plusieurs investissements majeurs dans l'aluminium. Essor qui s'est maintenu à la hausse avec la saga entourant la vente d'Alcan à des intérêts étrangers, le renouvellement de plusieurs conventions collectives ainsi que les incertitudes entourant les aides fiscales consenties aux régions ressources. Dans le cadre des campagnes électorales entourant cette période, les politiciens de toutes allégeances prennent également position face à l'avenir de la Vallée de l'aluminium.

La très grande majorité des petits événements observés eurent lieu à Ville de Saguenay et possèdent un rayonnement régional. Par contre, une dizaine de ceux-ci se sont déroulés dans d'autres villes du Québec, notamment des congrès et symposiums, alors que deux petits événements furent tenus en Europe.

Nous les avons classifiés dans une dizaine de catégories. La catégorie qui contient les conférences, cercles de presse, annonces et débats à la radio vient en tête avec plus du tiers des petits événements observés au cours de la période. Loin derrière, on retrouve les congrès, forums, symposiums, salons et expositions annuelles $(12,5 \%)$ et des assemblées et audiences publiques $(12,5 \%)$. Une fois sur dix, les petits événements prennent la forme de divers lancements. On dénombre onze petits événements dans la catégorie des comités, groupes de travail, rencontres ou réunions. Plus marginalement, existent des pétitions, des manifestations, des missions internationales ainsi que des campagnes de promotion.

\section{Graphique 1}

\section{Répartition des petits événements selon leurs instigateurs dans la Vallée}

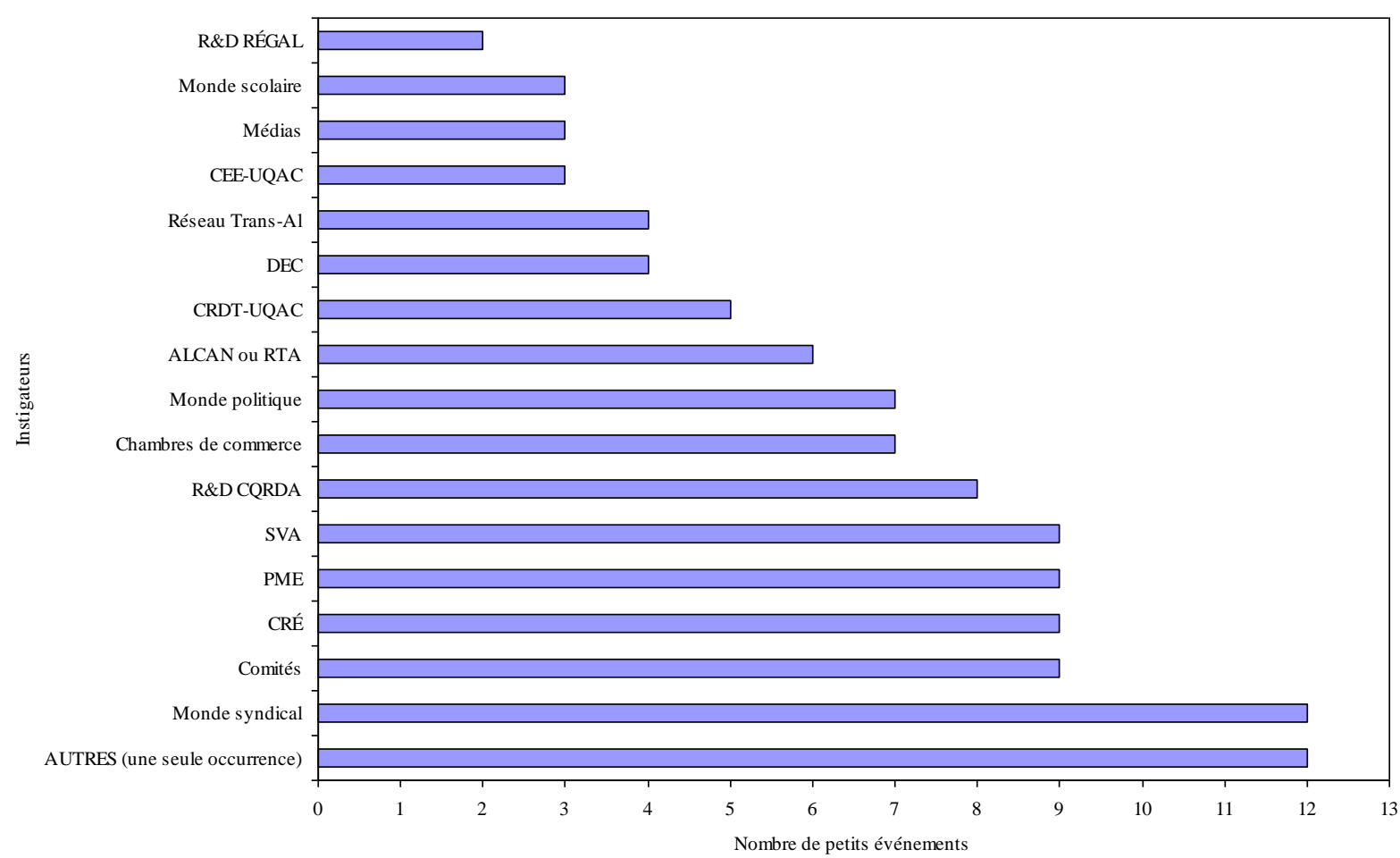

Le graphique 1 permet de constater que plusieurs instigateurs différents s'inscrivent à l'origine des petits événements dans la Vallée, dont voici les principaux. Le monde syndical occupe la première place ayant initié une douzaine de petits événements. Plusieurs 
comités se sont également impliqués dans l'organisation de neuf petits événements. Bien qu'elles animent des Cercles de conférence, on constate que les Chambres de commerce se laissent désormais tirer l'oreille face à leur rôle traditionnel d'organisatrices de petits événements. Rôle d'animation qui s'avère bien

\section{Les réseaux}

Contrairement aux regroupements, les réseaux n'impliquent pas une entente formelle entre les acteurs qui les tissent. Les réseaux véhiculent des interactions dans un but général de diffusion de l'information et de transfert des connaissances selon un esprit de libre circulation hors des relations marchandes. Les liaisons de réseautage sont généralement basées sur une relation de confiance entre les acteurs, éventuels partenaires. L'aboutissement normal du réseautage mène à l'apprentissage collectif.

Pour tenter de cerner le réseautage sous l'angle des liaisons générées dans la Vallée de l'aluminium, nous avons exploré à nouveau les journaux locaux via la banque de données "Biblio-Branchée » disponible à l'UQAC. Nous avons dénombré tous les articles qui mentionnaient des liaisons entre au moins deux acteurs distincts à travers les composantes du système productif de la Vallée (figure 3). Nous n'avons évidemment aucune prétention à saisir la qualité du réseautage et des contenus, ni des retombées concrètes.

Le tableau 2 qui illustre nos observations, les zones en blanc font état de l'absence de lien entre certains acteurs. Les zones en gris représentent les combinaisons où l'on dénombre plus d'une dizaine d'articles qui traitent de liaisons, peu importe les acteurs relevé cependant par l'UQAC et ses tentacules de R\&D. Finalement, en considérant le modèle utilisé (figure 3) pour l'analyse des petits événements dans la Vallée, on constate la faible présence du monde de la finance, de Promotion Saguenay, des CLD, des SADC, des gestionnaires d'infrastructures.

impliqués. Certains acteurs du secteur de l'aluminium sont ainsi mis en évidence par leurs nombreux liens tissés avec l'ensemble des autres acteurs de la Vallée. Les zones en italiques représentent chacune des composantes retenues par notre modèle d'analyse. Cette façon de traiter nos données permet de voir si les liens sont effectivement tissés serrés au sein d'une même composante et de saisir qui assure le leadership. Soulignons que nous n'avons aucune donnée pour documenter la composante concernant les infrastructures dans la Vallée.

Nous constatons que le système productif de la Vallée de l'aluminium comporte dans son ensemble quelques forces majeures sous l'angle du réseautage. En regard de notre modèle (figure 3), la composante concernée par les entreprises (Rio Tinto Alcan et PME) et celle contenant les équipementiers apparaissent en liaisons intimes formant de fait le cœur du réseautage dans la Vallée. Le domaine de la recherche semble également jouer un rôle important, plus précisément le CQRDA et le CTA-CNRC, mais également le CRDA. Au sein des organisations, ce sont la SVA, le Réseau Trans-Al et la CRÉ qui ont développé le plus de liens avec les autres acteurs du milieu. Finalement, l'UQAC joue un rôle clé dans le domaine de la formation. 


\section{Tableau 2}

\section{Liaisons issues du réseautage dans la Vallée}

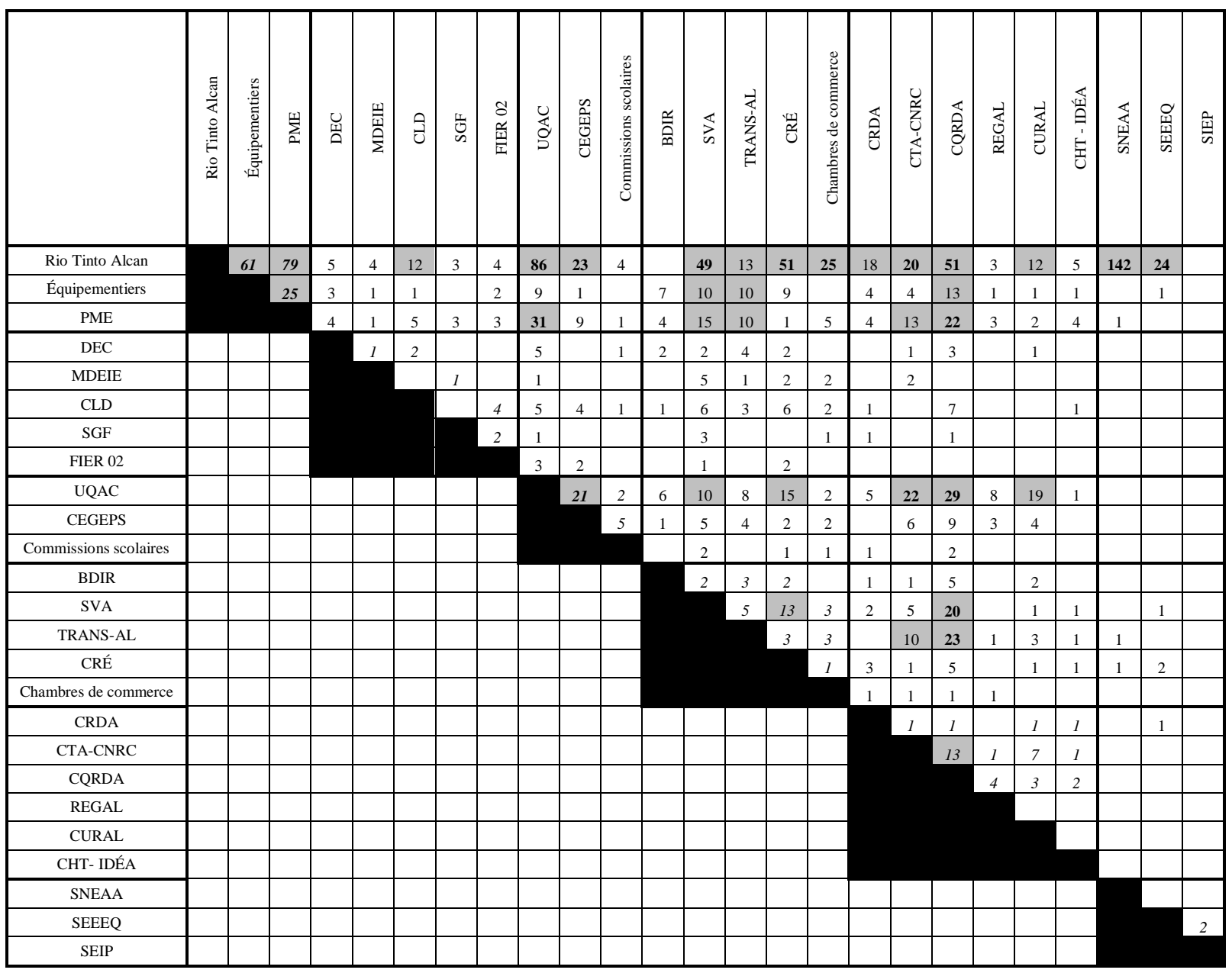

Source : Compilation personnelle à partir de la banque de données Biblio-branchée de l'UQAC

Des faiblesses à propos du réseautage dans la Vallée sont illustrées par l'analyse de nos données. Dans les liaisons, les syndicats du secteur de l'aluminium ne sont associés de près qu'à Rio Tinto Alcan, sans pratiquement tisser de liens formels avec les autres composantes du système productif de la Vallée de l'aluminium. Cela s'explique peut-être par la période d'observation qui correspondait à une négociation importante qui laissait peu de temps à d'autres types de relations. De plus, la composante concernée par le financement représente, dans son ensemble, un maillon faible du système avec de rares liens tissés avec d'autres composantes du réseau, puisque seuls les CLD ressortent un peu du lot avec quelques actions collectives en liaison à Rio Tinto Alcan. Signalons finalement à ce chapitre que pour diverses raisons, certaines interactions nous échappent puisque n'ayant pas fait l'objet de diffusion dans les médias locaux. 


\section{Les catalyseurs du milieu}

Existe-t-il des catalyseurs dans Vallée de l'aluminium ? On peut examiner cette question selon deux points de vue: soit celui des organismes, soit celui des individus.

En observant la mission et les objectifs poursuivis par les nombreuses organisations impliquées dans le soutien général à l'innovation dans la Vallée, plusieurs indications par des mots-clés (collaboration, coopération, concertation, partenariat, maillage, réseautage, synergie, ententes) nous permettent d'avancer qu'ils participent à la catalyse du milieu. Nous vous faisons grâce du détail de nos observations par manque d'espace, mais il ressort que plusieurs acteurs désirent jouer un rôle de catalyseur dans le secteur de l'aluminium. Certains visent la synergie à l'intérieur de leur propre sphère d'action, en recherche par exemple, avec parfois une ouverture sur le monde. D'autres ont une vision plus élargie et désirent créer des liens entre deux ou trois des composantes du système productif de la Vallée de l'aluminium. Mais aucun des acteurs ne s'identifie explicitement comme catalyseurs, médiateurs ou intermédiaires dans le processus d'innovation. Selon nos observations cependant, trois organisations agissent clairement comme des catalyseurs dans la Vallée pendant la période retenue, soit la SVA, le BDIR-RTA et DEC. À celles-ci, il faut ajouter le Comité d'orientation de la carte routière technologique canadienne de la transformation de l'aluminium qui a œuvré en véritable catalyseur en 2006. D'autres encore agissent dans cet esprit de catalyse sans toutefois s'y consacrer totalement.
Du côté des individus, l'observation des journaux locaux et aussi la composition des conseils d'administration des diverses organisations dans la Vallée nous a permis d'identifier huit personnes qui interviennent comme catalyseurs, œuvrant à plusieurs niveaux sur quatre ou cinq instances différentes.

Le tableau 3 indique que les catalyseurs $\mathrm{C} 1$ à C8 sont particulièrement actifs au sein de trois des composantes de R\&D de la Vallée. Plusieurs comités intersectoriels représentent aussi des lieux de catalyse par excellence.

Notons que la moitié des catalyseurs identifiés dans la Vallée ont siégé sur le Comité d'orientation de la carte routière technologique canadienne de la transformation de l'aluminium en 2006. Quatre catalyseurs font aussi partie du Comité d'orientation du REGAL qui a pour mission d'assurer la synergie entre les scientifiques, les besoins réels en évolution dans les industries, les différents représentants gouvernementaux et la société québécoise. Plusieurs se côtoient régulièrement au sein du CQRDA.

Terminons cette rubrique en soulignant que nous n'avons aucunement la prétention de présenter un tour complet de la question relative aux catalyseurs de la Vallée. Loin s'en faut ! Des analyses plus poussées à partir d'observations plus fines nous permettraient sans doute d'y voir plus clair, notamment par une étude des carnets d'adresses électroniques, des agendas d'affaires, des curriculums vitae, des procès verbaux. Mais ceci dépasse largement ici le cadre la présente analyse de type exploratoire. 


\section{Tableau 3}

\section{Répartition de huit individus catalyseurs dans la Vallée}

\begin{tabular}{|c|c|c|c|c|c|c|c|c|c|c|}
\hline COMPOSANTES & RAYONNEMENT & LIEU D'IMPLICATION & C1 & C2 & C3 & C4 & C5 & C6 & C7 & C8 \\
\hline ENTREPRISES & International & Alcoa & 1 & & & & & & & \\
\hline AUTRES & National & Comité d'orientation de la carte routière technologique & 1 & 1 & 1 & 1 & & & & \\
\hline FINANCEMENT & National & DEC & & & & 1 & 1 & & 1 & \\
\hline ORGANISATIONS & National & $\mathrm{ACA}$ & 1 & & & 1 & & & & \\
\hline RECHERCHE & Provincial & REGAL & 1 & 1 & 1 & & & & 1 & \\
\hline RECHERCHE & Provincial & CQRDA (différentes unités) & 1 & & 2 & & & 4 & & \\
\hline AUTRES & Provincial & Comité sectoriel de main-d'œuvre en métallurgie & & & & & 1 & & & \\
\hline ORGANISATIONS & Régional & CRÉ 02 (c.a. et comité de maximisation) & & & & & 1 & & & 2 \\
\hline ENTREPRISES & Régional & PME & & 1 & & & & & 1 & \\
\hline ORGANISATIONS & Régional & SVA & & & & 1 & & & & \\
\hline ORGANISATIONS & Régional & Trans-Al & & 1 & & & & & & \\
\hline RECHERCHE & Régional & CURAL & & & & & & & 1 & \\
\hline FINANCEMENT & Régional & CLD & & & & & & & & 1 \\
\hline AUTRES & Régional & Conseil régional de concertation et de développement & & & & & 1 & & & \\
\hline \multirow[t]{2}{*}{ ORGANISATIONS } & Local & Ville de Saguenay & & & & & & & & 1 \\
\hline & & NOMBRE TOTAL D'INSTANCES & 5 & 4 & 4 & 4 & 4 & 4 & 4 & 4 \\
\hline
\end{tabular}

\section{Conclusion}

L'interaction à finalité d'innovation (figure 2) s'avère belle et bien présente dans la Vallée de l'aluminium, avec ses regroupements, ses nombreux petits événements, ses réseaux et ses catalyseurs. Le nœud central de ce système productif territorial s'inscrit dans la puissante position de la multinationale Rio Tinto Alcan. Compagnie qui malgré son autonomie financière, technologique et matérielle, a su établir des liens avec la plupart des partenaires $\mathrm{du}$ milieu (équipementiers, R\&D, services spécialisés, éducation et formation, PME, organisations de soutien) afin de faire progresser ses intérêts corporatifs, soit la production d'aluminium primaire dans un contexte de concurrence mondiale.

On observe également dans la Vallée la présence de plusieurs autres «têtes de réseaux », notamment le Réseau Trans-Al,
CTA-CNRC, CQRDA, UQAC, certains comités, dont les intérêts s'inscrivent au pluriel, soit les $2^{\mathrm{e}}$ et $3^{\mathrm{e}}$ transformations de l'aluminium, les subventions de recherche, les publications scientifiques, le financement des initiatives, l'innovation, la formation professionnelle, le développement économique, etc. Dans ce système d'interaction et d'apprentissage qui sied dans la Vallée, signalons aussi l'absence ou la faible présence d'acteurs pourtant importants en principe, notamment les CLD, les SADC, les organismes de financement, les entreprises phares, Promotion Saguenay, les syndicats, la SFR 02, les Chambres de commerce, certains regroupements, les organisations de formation professionnelle, qui ont tous des objectifs à poursuivre en regard de l'industrie de l'aluminium. 
Tous ces nœuds bien actifs en matière d'interaction apparaissent considérablement repliés sur leur propre réseau - égo. Replis corporatifs et individuels à degrés divers qui limitent la formation d'un nécessaire « réseau de réseaux » entre les différents acteurs dans la Vallée. Ce qui conforte les postures divergentes autour de finalités communes à poursuivre en principe. La diversité des intérêts défendus et des objectifs ciblés par les principaux acteurs indépendants mais aussi interdépendants, contraint la dotation d'une vision globale partagée de leur Vallée et de sa mission en matière d'innovation. En réalité selon le modèle interactif (figure 2), l'apprentissage collectif à l'innovation dans la Vallée s'avère clairement dominé par RTA et son Bureau industriel ainsi que par les centres de $R \& D$. Situation tout à fait singulière et insatisfaisante au sein de laquelle la SVA joue heureusement un rôle d'ouverture vers certains autres acteurs certes moins imposants mais néanmoins essentiels au processus de conception de produits, de services et de procédés.

Cette importante faiblesse de la Vallée en matière d'interaction et d'apprentissage collectif n'est aucunement insurmontable. Nous en avons pour preuve la méthode utilisée récemment par le Comité d'orientation de la carte routière technologique canadienne de la transformation de l'aluminium.
Ce comité a bel et bien illustré une voie originale et constructive en matière de recherche réellement collective de solutions innovantes pour l'ensemble de la Vallée. Ce qui illustre que l'apprentissage collectif autour de finalités communes d'intérêt général pour la Vallée s'avère possible à accélérer. C'est-àdire qu'une interaction inter - réseaux semble accessible et atteignable, à partir de la base interactive riche et disponible. Ne manque que la volonté collective pour supporter un nouveau leadership de cohésion globale et de synergie dans l'apprentissage au sein de la Vallée. Définir collectivement une véritable vision globale qui pourrait conduire vers l'adoption d'objectifs communs bien ciblés, représente à notre avis l'étape numéro 1 dans cette voie de l'innovation dans la Vallée. Une telle opération de visionnement collectif devrait nécessairement être accompagnée par l'animation continue d'une série de «cercles de créativité » capables de mobiliser entièrement les expertises du milieu et les expertises scientifiques autour de problèmes, de menaces, de contraintes, d'occasions dans un esprit de solutions.

En conséquence, nous recommandons la mise en œuvre d'un Forum permanent qui pourrait agir comme catalyseur général de l'apprentissage collectif, de la créativité et de l'innovation dans la Vallée de l'aluminium.

\section{Notes et références}

1 Voici les adresses électroniques de ces deux sites : LE BULLETIN RÉGIONAL (LBR) : http://www.lbr.ca; BIBLIO-BRANCHÉE : http://www.biblio.eureka.cc.sbiproxy.uqac.ca/WebPages/Search/Results.as 


\section{LEXIQUE}

ACA : Association canadienne d'aluminium

BDIR-RTA : Bureau de développement industriel régional de Rio Tinto Alcan

C.C. : Chambre de commerce

CEE-U QAC : Centre d'entreprenariat et d'essaimage de l'UQAC

CEGEP : Collège d'enseignement général et professionnel

CHT-IDÉA : Centre de haute technologie (maintenant IDÉA Innovation PME)

CLD : Centre local de développement du Québec

CQRDA : Centre québécois de recherche et de développement sur l'aluminium

CRDA-RTA : Centre de recherche et développement Arvida de Rio Tinto Alcan

CRDT-UQAC : Centre de recherche sur le développement territorial de l'UQAC

CRÉ 02 : Conférence régionale des élus du Saguenay - Lac-Saint-Jean

C.S. : Commission scolaire

CTA-CNRC : Centre des technologies de l'aluminium du Conseil national de recherches Canada

CURAL-UQAC : Centre universitaire de recherche sur l'aluminium de l'UQAC

DEC : Développement économique Canada

DRH Canada : Direction des ressources humaines Canada

FIER 02 : Fonds d'intervention économique régional du Saguenay - Lac-Saint-Jean

LBR : Le Bulletin régional du Saguenay - Lac-Saint-Jean

LSJ : Lac-Saint-Jean

MDEIE : Ministère du Développement économique, innovation et exportation du Québec

PME : Petites et moyennes entreprises

R-D : Recherche et développement

REGAL : Regroupement aluminium

RTA : Rio Tinto Alcan

SADC : Société d'aide au développement des collectivités

SEEEQ : Syndicat des employés d'énergie électrique Québec

SEIP : Syndicat des employés des installations portuaires

SFR 02 : Société des fabricants régionaux du Saguenay - Lac-Saint-Jean

SGF : Société générale de financement du Québec

SLSJ : Saguenay - Lac-Saint-Jean

SNEAA : Syndicat national des travailleurs d'Alcan à Arvida

SVA : Société de la Vallée de l'aluminium

TRANS-AL : Réseau Trans-Al

UQAC : Université du Québec à Chicoutimi

VALLÉE : Vallée de l'aluminium au Saguenay - Lac-Saint-Jean 


\section{Publicité}

\section{Affaires municipales et régions}

